\title{
eSport vs irlSport
}

\author{
Christopher McCutcheon $^{1(\mathbb{*})}$ (D), Michael Hitchens ${ }^{2}$ (D), and Anders Drachen ${ }^{3}$ (iD \\ 1 Torrens University Australia, Leura, Australia \\ christopher.mccutcheon@laureate.edu.au \\ ${ }^{2}$ Macquarie University, Macquarie Park, Australia \\ michael.hitchens@mq. edu . au \\ ${ }^{3}$ Digital Creativity Labs, University of York, York, UK \\ anders.drachen@york.ac.uk
}

\begin{abstract}
This paper examines in-real-life (irl) sport and eSports in an attempt to clarify the definition of eSport. The notion of physicality and embodiment are central to the need for clarity in understanding of what eSports are and whether they are sport or some other activity. By examining existing definitions of eSport and irlSport we can identify the similarities and differences between these activities. Methodologically the paper uses the philosophical process of critical thinking and analysis to examine the various approaches taken to defining both eSport and irlSports. Our aim is to highlight the inherent problem of the definition of eSports and irlSports (and the privileging of the term sport as it currently applies only to irlSports). We find that eSports are sports and that the definition of sport should be expanded to include sub-categories of irlSports and eSports.
\end{abstract}

Keywords: eSport $\cdot$ irlSports $\cdot$ Competitive video games $\cdot$ Embodiment Virtual play spaces

There are few words in the English language which have such a multiplicity of meanings as the word sport [1].

eSport has similar multiplicity, for example:

eSports commonly refer to competitive (pro and amateur) video gaming that is often coordinated by different leagues, ladders and tournaments, and where players customarily belong to teams or other "sporting" organizations which are sponsored by various business organizations [2].

and

"Esports is computer games played in a competitive environment" [3].

A. Drachen-Part of this work was conducted in the Digital Creativity Labs (www.digitalcreativity.ac.uk), jointly funded by EPSRC/AHRC/InnovateUK under grant no EP/M023265/1.

(C) The Author(s) 2018

A. D. Cheok et al. (Eds.): ACE 2017, LNCS 10714, pp. 531-542, 2018.

https://doi.org/10.1007/978-3-319-76270-8_36 


\section{Introduction}

Throughout the history of humanity's attempts to understand sport from a theoretical or philosophical perspective, the theorists and philosophers have in general taken to view sport in terms of physicality. As eSports emerge from the niche 'gamer-nerd' segment to be embraced by a wider, mainstream audience and culture there is an increasing need to clarify our understanding of what eSports are and how they relate to traditional embodied sports or 'in-real-life' or irlSports ${ }^{1}$.

Clarity is required as the current definition of sport does not take into consideration the discipline of eSports. Decisions are being made, laws enacted, resources invested without a functioning definition or anything but a lay understanding of what eSports actually are. The notion that physicality is central to the concept of irlSport has never really been questioned. The current focus upon physicality creates some interesting challenges when we attempt to understand how eSports match up or compare to traditional in-real-life (irl) sports. In this paper we will attempt to rectify part of the problem by investigating the various definitions that we have of eSports and irlSports ${ }^{2}$. We argue that the definition of sport must change to accommodate the rapid growth of eSports. Ultimately however, it is open for debate whether eSports are indeed sports, or some other category of sports-like or sportive competitive behaviour.

eSports are considered to be, in general, a competitive approach to computer games. For example, Hamilton et al. [4] defined eSports to be "the high-level play and spectating of digital games". Several other authors and theses agree in general terms with the simple definition of eSports as competitive computer games [5,6]. There are several issues with the current definition of irlSports which we believe are problematic. Any of these issues are sufficient to demand a new look at how irlSports and eSport are defined, and the relationship (if any) between them.

Some of the identified issues with the current (philosophical) definition of irlSport which have a bearing or impact upon the subsequent definition or understanding of eSport:

It is impossible to define an open system without reverting to arbitrary stipulation ${ }^{3}$. All sport activities are social systems, social systems are open to change and thus constitute open systems. Therefore sports are open systems and impossible to define without arbitrary stipulation. We contend that the notion of physicality is such an arbitrary stipulation.

The philosophical definition of sport was essentially set in its current form in 1985 . There has been little to no new investigation of our understanding of what sport really

${ }^{1} \mathrm{irl}=$ in real life. A term used by gamers and other online populations to mean anything that happens in real life, or outside of the game/MMO/internet environment.

2 Hemphill started the inquiry in 2005 with his work, Cybersports. In that paper he questioned the privileging of the 'real' over the virtual. Part of the underlying thesis of this paper harks back to that investigation of Hemphill's - to question the othering of the virtual as not real, and to challenge the notion that sport must be likewise grounded in the physical or the 'real'.

${ }^{3}$ Stipulation; the practice of defining any object or thing by declaring it so. Stipulation is not a desired method of philosophical inquiry or a preferred definitional approach as it can lead to arbitrariness, and the fuzzy edge of a set becomes a problematic case for any stipulation. 
is (ontology) since. Therefore, the emergent sportive practice of eSport, which came after the development of the current prevailing definition, does not fit comfortably within the current definition of sport, and as such leaves that current definition outside of the necessary and sufficient conditions to ontologically describe sport in all of its forms.

Physicality is a core element of the currently accepted definition of sport. Physicality itself is problematic in terms of monist embodiment (no mind/body split) and distributed communication/embodiment systems.

Online, distributed personality (leaky body concept [7]), genetic modification technology, body modification, prosthetics, anthropomorphism and nanotechnology (not an exhaustive list by any measure) all muddy the concept of physicality, humanity and embodiment. Again leaving the current definition of sport on shaky grounds - eSports adds further to the problematic definition that sports are at their core games with the skillful exercise of physicality as their distinguishing feature from the aforementioned said core class of games.

This paper will examine eSports by, in part, examining the (philosophical) literature on irlSport and hopes to determine a forward pathway for determining a method of definition or class-object analysis for the irlSports-eSport juncture.

Methodologically, the paper uses the philosophical method of critical thought and analysis. It examines the underlying definitional approaches to eSport and irlSport and concludes that there are fundamental problems with the current approaches to defining both. By considering the role of physicality and outcomes in understanding eSport the paper suggests a potential avenue of approach to dealing with the problem, and identifying pathways for future work.

\section{2 eSport: Impact and Popularity}

eSports commonly refer to competitive (pro and amateur) video gaming that is often coordinated by different leagues, ladders and tournaments, and where players customarily belong to teams or other "sporting" organizations which are sponsored by various business organizations [2].

Hamari and Sjöblom [2] stated that eSports are becoming one of the most rapidly growing media in the world; that growth being driven by the increasing number of online games and broadcasting channels such as pod and vod casting, youtube and twitch streaming just to name the more obvious and accessible. This is supported by Heaven [8] who indicated that better video-streaming and internet speeds are enabling greater engagement with eSports.

Meanwhile Sjöblom et al. [9] indicated that eSports streaming is at the centre of a major shift in the broadcast media landscape. An example of this is provided by the July 2017, Sydney leg of the Overwatch World Cup qualifiers; which we believe were the first eSports matches broadcast on Australian free to air television ${ }^{4}$.

eSport has made its way into popular culture and is being recognized by mainstream society. According to Hamilton, Kerne and Robbins [4] "The Global StarCraft League (GSL) finals at Blizzcon 2011...engaged 25,000 co-located and 300,000 online

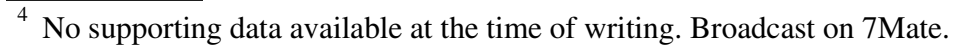


viewers." Snider [10] claimed that in 2013 League of Legends (a popular eSport) had 32 million players each month.

Similarly, Wingfield [11] indicated that (as at 2014) an estimated 70 million people watch eSports online ${ }^{5}$ and the 2013 League of Legends championship achieved an online viewership of 8.5 million; compared to the 7.2 million viewers of the ITV broadcast of the FA Cup Finals (Wigan vs Manchester City) in the same year [12].

eSports leagues are a central feature of the eSports communities and growth of the phenomena. Multiple eSports leagues and competitions have emerged all over the world - ESL Gaming, Major League Gaming, World Cyber Games (defunct), and more recently, the League of Legends and Overwatch World Cups [13-18].

Coats and Parshakov [19] analyzed the prize pools in eSports tournaments, finding that eSports players are risk averse and advising that there should be a larger spread of the prize pool to incentivize players. Whilst Hollist [20] argued that there is a need for regulation of labor relations in eSports as current laws are inadequate to properly regulate eSports in regards to employer-employee relations and employment conditions and employee (player) health. There is a considerable amount of interest in eSports from various sectors of the academy - commercial gaming [21], grassroots eSports communities [22], eSports and streaming content [9, 23], nationality [24], training and physicality [25] and so on.

To sum up, in the words of Hamari and Sjöblom [2], "During recent years, eSports (electronic sports) have become one of the most rapidly growing forms of new media driven by the growing provenance of (online) games and online broadcasting technologies".

It is clear that eSports have become significant in both business and societal terms, and therefore it is important to develop a clear definition or understanding of what eSports actually are.

\section{3 eSports: Current Definitions and Approaches}

e-sports, a catchall term for games that resemble conventional sports insofar as they have superstars, playoffs, fans, uniforms, comebacks and upsets. But all the action in e-sports occurs online, and the contestants hardly move [26].

There are an astounding number of eSports definitions in both the academic and nonacademic literature. Unfortunately all of them are stipulative and have not been tested; and as such cannot function as definitions from a philosophical point of view. However, they generally suffice for a 'working definition' point of view.

Hemphill's definition, as a sport philosopher, is arguably the closest there is to an acceptable eSport definition [27]: "electronically extended athletes in digitally represented sporting worlds." (p. 199). Hemphill updated and extended this in 2015 '... contrary to the claim about them being virtual or merely games, sport-themed computer games that involve human immersion and skillful, physical interactivity can be considered sport, at least in the classic formulation of sport as the demonstration of physical prowess in a game' [28].

\footnotetext{
${ }^{5}$ Wingfield did not provide information on the timeframe over which this number was accrued.
} 
Wagner [6] defined eSports as "an area of sport activities in which people develop and train mental or physical abilities in the use of information and communication technologies." The fundamental problems associated with this definition are due to the overly broad nature of the foundation upon which Wagner laid it. ${ }^{6}$

Hamari and Sjöblom [2]: "a form of sports where the primary aspects of the sport are facilitated by electronic systems; the input of players and teams as well as the output of the eSports system are mediated by human-computer interfaces" [2]. A clear development upon Wagner. Martončik [29] follows a similar train of thought.

Alternatively Hutchins [30] suggests that the eSport model is nothing more than a template overlaid upon the traditions, mores and performative culture of mainstream sports broadcasting. The intention of such being to tap into the continuity of broadcast sports, and in doing so be accepted as a sport by virtue of being called a sport and appearing like a sport. Or to put it another way if it walks like a duck, talks like a duck and looks like a duck, it must be a duck; Essentially Wittgenstein's family resemblance model.

Karhulahti [21] neatly summed up the working definitions as:

With nuance, they all perceive esport through two criteria: technological specificity (computers, cyberspace, electronics) and advanced competition (athleticism, professionalism, sport). These criteria are directly connected to the videogame culture so that esport is recognized as an "extension of gaming."

Therefore the working definition can be distilled down to two generic views of eSport; eSport is computer mediated competitive sportive or sport-like behavior. To clarify:

- Sportive - has the features of a sport according to the Suits-Meier formulation of sport in the philosophy of sport literature, but does not necessarily 'look' like a traditional sport. E.g. League of Legends [31] or Overwatch [32]

- Sport-like - a digital representation of an irlSports which on the surface appears to be a sport but may not be categorized as a sport according to the Suits-Meier formulation. For example, FIFA 17 [33].

The fundamental problem however, is that there has not been an examination of the metaphysical or ontological foundations underlying pronouncements on what eSports are. There have been stipulated, but relatively sensible working definitions made (see all of the above literature). Those definitions, for the most part have functioned well enough for researchers looking at issues surrounding eSports (regulation, player management, prize money, gambling, game design and mechanics and so on and so forth) but to date, no single clear investigation into the nature of eSports, or their relationship to irlSports, or to the foundational disciplines of games, play and ludology (which will have to be left to further study due to space restrictions here). Therefore, it is difficult, and somewhat irresponsible, to rely on lay, or untested definitions of the concept of eSports, and/or their relationship to irlSports.

\footnotetext{
${ }^{6}$ Wagner bases his definition of eSports on that of the work of Tiedemann [53], which seems overly broad; so broad as to essentially include all human activity as sport. Further, Tiedemann does not take into consideration the generally accepted Suits-Meier definition of sport.
} 


\section{Defining eSports or Re-defining irlSport? Why not Both!}

It may not be possible to come to a unified and acceptable (analytic/essentialistic philosophical) definition of sport that will have the necessary and sufficient conditions to encompass both eSport and irlSports. Therefore the next two viewpoints may have long term merit in the evolution of an acceptable understanding of eSports.

Karhulahti [21] considered eSport not from the competitive and technology leveraged point of view that many authors have taken, but from the economic 'pay-for-play' point of view, and introduced the idea of "Executive Ownership" (p. 46), where the owner of the intellectual property - i.e. the game company that develops and maintains the game, servers and 'playing fields', has ultimate power and ownership over when and even how the game/sport is played. Karhulahti further suggests that eSport be described as economic Sport, rather than electronic. Karhulahti points out that her view of economic eSport should encompass any commercial game (with attached Executive Ownership structure - holder of absolute power in regards to property rights over the game) that has a competitive, social and instructional structure surrounding it.

Interestingly, Karhulahti's definition does have a remarkable similarity to Bernard Suits' $[34,35]$ original definition of sport, sans the physicality component (more on this later).

A second alternative viewpoint is that of Wittgenstein's [36] notion of family and community that can easily be applied to a definitional approach to eSports.

Wittgenstein claimed there cannot be an essentialistic ${ }^{7}$ definition of irlSports because there are no necessary and sufficient conditions which are broad enough to cover all aspects but specific enough to limit out non-sport elements.

If we accept Wittgenstein's thesis then it is basically impossible to essentially define the product of any social (human defined) system. Therefore, sport, and presumably eSport, as the product of social systems, are both open sets and therefore cannot be defined $^{8}$. Thus any attempt to close the set is arbitrary and therefore stipulative; which ultimately defeats the purpose of defining the fundamental essence of eSport and irlSports.

Wittgenstein offered a different way in which we can view of eSport/irlSport; that sport may be characterised upon the idea of family resemblance or commonality. Under this approach, eSports are sports because of the broad family resemblance between them and irlSports. The family name (eSports are in fact named eSports) automatically making them a sub-set of the class sport. It is all in the name, similar things share a similar name.

In addition there is the socially accepted role that eSports plays in the eSports community. eSports fulfil the same social role as irlSports within the gamer community. As such eSports can be considered to be equivalent to irlSports for the social niches that accept eSports as sports. This is known as the equivalency clause:

\footnotetext{
${ }^{7}$ Essentialistic is taken to mean any underlying essential or fundamental truth or knowing of a thing.

${ }^{8}$ However, a method (class membership) may be substituted instead of definition. To be honest we are delving into the semantics/deconstruction of the definition of 'definition'; which is somewhat out of the scope of this paper.
} 
For example, overheard at the Sydney leg of the 2017 Overwatch World Cup Cosplayer 1, whilst enthusiastically cheering after the Australian team win over Japan, turned towards Cosplayer 2 and yelled joyously, "Now I get sport!" [37].

Evidence of an eSports community is provided by Kozachuk, Foroughi and Freeman [38] who described the eSport as being in a state of "drastic growth", with increasing numbers of player and competitions and "millions of spectators" globally. Likewise Freeman [39] added that, "esports players extremely emphasize the sense of community, belongingness, cohesion, and comradeship among them."

The final word here goes to Gunatilaka, [40] commenting on the Sydney leg of the 2017 Overwatch World Championship:

To anyone in doubt of whether esports should be considered a sport, take it from me - it is sport and it deserves to be. These tournaments feel exactly the same as packed out footy games. Forget the fact that compared to traditional sports, esports has little physical exertion. The amount of teamwork, training, skill and dedication required to compete at this level is legitimate.

\section{What is irlSport?}

The current presiding definitions of games, play and sport fail to take into consideration the changing nature of the online or virtual world and the technology and culture that drives, sustains and surrounds these worlds. The terrain has significantly changed since Klaus Meier [41, 42] suggested some changes to Bernard Suit's [35, 43-47] definition of games and sport in 1989. It has become important to re-examine the definition of sport and performance and, indeed, the western cultural understanding of what a sport is in light of the development and impact of eSports.

Any such "definition" offered must, of course, be founded in an academic discourse, discipline or assumption; and as previously evidenced, there are many relevant and acceptable discourses within the sport, recreation and physical education sphere [48, $49]^{9}$. Each discourse presents a different viewpoint and aims to achieve a different agenda. In the case of sport philosophy, the discipline inherited its foundational discourse and understanding of physicality, humanity and activity primarily from physical education, and has never really challenged those inherited basic embodiment assumptions; arguably remaining basically essentialist throughout its existence as a distinct discipline.

We contend that the generally accepted definition of sport provided by Suits and Meier does not adequately cover eSports or virtual/online and distributed playing fields. By relying upon the concept of physicality, the current generally accepted understanding of irlSport excludes many eSport instantiations and practices, and provides important gatekeeper and policy development organizations (such as government funding agencies) with an incorrect philosophical and in-practice foundation on which they base their activities.

\footnotetext{
${ }^{9}$ Blumenfeld noted these different points of view in terms of play. He indicated the difficulty in determining exactly what play is stems from "...the different points of view which must be, but have not always been, clearly distinguished..." [48].
} 
Osterhoudt [50] and Paddick [51] as prime examples of the state of the discipline, emphasize physicality as "a necessary component (and intrinsic good) of sport" [52].

\subsection{The Suits/Meier Formulation for Sport}

"Games are the voluntary attempt to overcome unnecessary or gratuitous obstacles" [27].

The Suits/Meier formulation is generally accepted within the discipline of the philosophy of sport as the standard account for a definition of sport. This definitional approach has two distinct phases. The definition of games, which form the basis for the definition of sports.

The Suits $[35,47]$ formulation of games can be summarized as follows, games are:

- Goal oriented

- Rules based

- Where the rules prohibit the use of more efficient means over less efficient means, and,

- The rules are accepted just because they make the activity possible

Suits $[34,35]$ continued to define sport in such a manner:

A game is also a sport if:

- It is a game of skill,

- The core skill is physical,

- The game has a wide following, and

- The following has achieved a certain level of stability

This is the currently accepted “definition" of sport (Meier's modification of Suits):

... all sports are indeed games. That is, a game may also correctly be termed a sport if it possesses the additional characteristics of requiring physical skill or prowess [our emphasis] to be demonstrated by the participants in the pursuit of its goal [42].

We claim that eSports meets all the aspects currently required of the definition of sport. A simple 'in your own head' analysis will confirm that eSports are games.

Further; eSports fit into the above definition of sport if we consider that physicality is inherent in any human embodied activity. That is to say we are all meat; thinking requires the physical brain to occur, therefore eSports are physical. Also, of course, using a controller or mouse and keyboard is also physical.

As previously evidenced, eSports demonstrate skillful participation and enjoy a wide following; adhering to Suits [35, 43-47] original definition of sport.

This permits an examination of eSports alongside irlSports. Are eSports 'just' sports? To answer, as sports are not 'just' games then no, eSports are not just sports. There is a difference between the two similar but related concepts.

What sets eSports apart from sports? When we remove the notion of physicality that traditionally defined the difference between sport and games, and nominally defines the difference between sport and eSport, we are left in the undesirable position that games, sport and eSport are all the same thing; which clearly they are not. 
Hamari and Sjoblom [2] question the 'location' of irlSports vs eSports and declare that irlSports exist within the 'real' world whilst eSports exist outside of the 'real' world inhabiting virtual computer mediated environments. However, phenomenology principles could easily argue that this is a false premise since, the computer mediated delivery systems themselves are both in the world and of the world and therefore, any virtual environment is automatically in the 'real' world by virtue of inheriting its realness from its very existence.

irlSports are practiced in an arguably different form of embodiment and reality to that of eSports. Inasmuch that if we accept, for conveniences sake, the notion that the irlSports play-space is both in and of the world, whilst the eSport play-space could be argued that it is primarily only of the world ${ }^{10}$.

We can also refer to the concept of the leaky body [7], if we can accept that an irlAthlete can have their body boundaries and identity extended beyond the physical instantiation and biological limitation. Then clearly it should also be acceptable to apply the leaky body concept to the practice of sport (not just the practitioner) and thus also extend sport beyond the 'physical' limitations to include unbound examples; eSport.

A further approach would be to simply extend the prevailing definition of sport to encompass eSport as a sub-set, much like the definition of games was extended to include sport. Therefore, conceivably, we can conclude that eSports are sports (they really are) but they're a particular type/category/subset of sport, with, as yet to be clearly determined or defined parameters.

\section{Where to Next?}

Ideally, sport as a whole should remove references to physicality, as it is arbitrary, limiting and ambiguous. Additionally, physicality seems less important to eSports practice, however, there are similarities between irlSports and eSport on this front, in that eSport does contain physicality (reaction time, fine motor skills etc.) as a central feature of success. Or to put is very plainly and simply; physicality matters to eSport but is not all important. Additionally there needs to be clarity around accepting or rejecting eSports as a category of sports.

Finally, we suggest that there is potential for conceptualizing all sport not just in terms of physicality but more importantly goal directed skillful embodiment, whilst still embracing Suits notion of unnecessary obstacles and the play-space. We would suggest that characterizing sport in terms of outcomes within the world or impact upon an environment rather than privileging physicality would be a place to start this investigation. Where:

- Skillful can mean intentional, with skill and practice, and predominately non-random.

- Embodiment can mean any form of authentic cognitive lived embodiment regardless of physical form.

- Goal directed can mean any form of intentional action seeking a (prescribed/ preferred) outcome; regardless of achieving the outcome.

\footnotetext{
$\overline{10}$ Although eSport must be in the world for it to exist.
} 
- Playspace can mean any place in which play, games and sports are instantiated. The play space may be real, virtual, conceptual or imagined, or any variant as yet unidentified.

Therefore, we may consider an overall category of sport containing the sub-categories of eSport and irlSports. Further, the sub-categories of eSport and irlSports can be differentiated from each other by the embodiment typically expressed during their execution or performance (physical and virtual) and the environments/play spaces that they typically inhabit. Additionally, a third category of sportive behavior may be identified in those sports that exhibit crossover features of both irlSports and eSport. Specifically those activities that use virtual reality and motion capture devices (and future devices of this ilk) to translate full-embodied physical actions into a virtual eSports play space.

Sport is more than just irlSports. irlSports does not encapsulate all sport and eSport is more than just competitive computer gaming.

\section{References}

1. Graves, H.: A philosophy of sport. Contemp. Rev. 78, 877-893 (1900)

2. Hamari, J., Sjöblom, M.: What is eSports and why do people watch it? Internet Res. 27, 211232 (2017)

3. Schubert, M., Drachen, A., Mahlmann, T.: Esports analytics through encounter detection. In: MIT SLOAN Sports Analytics Conference, pp. 1-18 (2016)

4. Hamilton, W., Kerne, A., Robbins, T.: High-performance pen + touch modality interactions: a real-time strategy game eSports context. In: Proceedings of the 25th Annual ACM Symposium on User Interface Software and Technology (UIST 2012), pp. 309-318 (2012)

5. van Ditmarsch, J.: Video Games as a Spectator Sport: How Electronic Sports Transforms Spectatorship (2013)

6. Wagner, M.G.: On the scientific relevance of eSports. In: International Conference on Internet Computing, pp. 437-442 (2006)

7. Shildrick, M.: Leaky Bodies and Boundaries: Feminism, Postmodernism and (Bio) Ethics. Routledge, London (1997)

8. Heaven, D.: Esports: Pro video gaming explodes with big prize pots. https:// www.newscientist.com/article/mg22329823.900-esports-pro-video-gaming-explodes-withbig-prize-pots/\#.VR9OzeEyRxI

9. Sjöblom, M., Törhönen, M., Hamari, J., Macey, J.: Content structure is king: an empirical study on gratifications, game genres and content type on Twitch. Comput. Hum. Behav. 73, 161-171 (2017)

10. Jenny, S.E., Manning, R.D., Keiper, M.C., Olrich, T.W.: Virtual(ly) athletes: where eSports fit within the definition of "sport". Quest 69, 1-18 (2016)

11. Wingfield, N.: In E-Sports, Video Gamers Draw Real Crowds and Big money. https:// www.nytimes.com/2014/08/31/technology/esports-explosion-brings-opportunity-richesfor-video-gamers.html

12. Plunkett, J.: BBC's FA Cup final coverage nets peak of nearly 9 million (2015). https:// www.theguardian.com/media/2015/jun/01/bbc-fa-cup-final-coverage-nets-peak-ofnearly-9-million

13. Blizzard Entertainment: Overwatch World Cup 2017. https://worldcup.playoverwatch.com/en$\mathrm{gb} /$ \#group-stage 
14. Riot Games: World Championships. http://www.lolesports.com/en_US/worlds/ world_championship_2016/schedule/elim/Quarterfinals

15. Major League Gaming: Call of Duty World League. http://www.majorleaguegaming.com/

16. World Cyber Games: About the World Cyber Games of the Past. http:// worldcybergames.com/

17. Australian eSports League: Welcome to eSports. http://ael.org.au/

18. Turtle Entertainment: ESL Gaming. https://www.eslgaming.com/

19. Coates, D., Parshakov, P.: Team vs. Individual Tournaments: Evidence From Prize Structure in eSports Basic Research Program (2016)

20. Hollist, K.E.: Time to be grown-ups about video gaming: the rising esports industry and the need for regulation. Ariz. Law Rev. 57, 823-847 (2015)

21. Karhulahti, V.-M.: Reconsidering esport: economics and executive ownership. Phys. Cult. Sport. Stud. Res. 74, 43-53 (2017)

22. Curley, A.J., Nausha, M.: Challenges and Best Practices in Supporting Grassroots eSports Communities, pp. 1-5 (2016)

23. Karhulahti, V.-M.: Prank, troll, gross and gore: performance issues in esport live-streaming. In: 1st International Joint Conference on DiGRA FDG, pp. 1-13 (2016)

24. Parshakov, P., Zavertiaeva, M.: Success in eSports: Does Country Matter? (2016)

25. Kari, T., Karhulahti, V.-M.: Do e-athletes move? A study on training and physical exercise in elite e-sports. Int. J. Gaming Comput. Simul. 8, 53-66 (2016)

26. Segal, D.: Behind league of legends, e-sport's main attraction. https://www.nytimes.com/ 2014/10/12/technology/riot-games-league-of-legends-main-attraction-esports.html

27. Hemphill, D.: Cybersport. J. Philos. Sport. 32, 195 (2005)

28. Hemphill, D.: Cybersport. In: Torres, C.R. (ed.) The Bloomsbury Companion to the Philosophy of Sport, pp. 346-348. Bloomsbury Publishing, London (2015)

29. Martončik, M.: E-Sports: playing just for fun or playing to satisfy life goals? Comput. Hum. Behav. 48, 208-211 (2015)

30. Hutchins, B.: Computer gaming, media and e-sport. In: TASA Conference 2006, Perth, Western Australia, pp. 1-9 (2006)

31. Riot Games: League of Legends (2009)

32. Blizzard Entertainment: Overwatch (2016)

33. Electronic Arts: FIFA 17 (2016)

34. Suits, B.: The trick of the disappearing goal. J. Philos. Sport. 16, 1-12 (1989)

35. Suits, B.: Tricky triad: games, play, and sport. J. Philos. Sport. 15, 1-9 (1988)

36. Wittgenstein, L.: Philosophical Investigations. Wiley-Blackwell, Chichester (West Sussex) (2009)

37. McCutcheon, C.: Personal recollection (2017)

38. Kozachuk, J., Foroughi, C.K., Freeman, G.: Introduction. In: Exploring Electronic Sports: An Interdisciplinary Approach, Proceedings of the Human Factors and Ergonomics Society Annual Meeting, pp. 2118-2122 (2016)

39. Freeman, G.: How, if at all, does eSports shape players' online and offline social/interpersonal relationships? In: Exploring Electronic Sports: An Interdisciplinary Approach, Proceedings of the Human Factors and Ergonomics Society 2016 Annual Meeting, p. 2119 (2016)

40. Gunatilaka, L.: Falling for eSports. https://www.facebook.com/notes/screenplay/falling-foresports-blog/2093700917323067/

41. Meier, K.V.: Performance prestidigitation. J. Philos. Sport. 16, 13-33 (1989)

42. Meier, K.V.: Triad trickery: playing with sport and games. In: Morgan, W.J., Meier, K.V. (eds.) Philosophic Inquiry in Sport, pp. 23-35. Human Kinetics, Champaign (1995)

43. Suits, B.: What is a game? Philos. Sci. 34, 11-17 (1967) 
44. Suits, B.: The elements of sport. In: Osterhoudt, R. (ed.) Philosophic Inquiry in Sport, pp. 48-64. Human Kinetics, Springfield (1973)

45. Suits, B.: The Grasshopper: Games, Life and Utopia. NonPareil, Boston (2005)

46. Suits, B.: Words on play. J. Philos. Sport. 4, 117-131 (1977)

47. Suits, B.: The elements of sport. In: Morgan, W.J., Meier, K.V. (eds.) Philosophic Inquiry in Sport, pp. 8-15. Human Kinetics, Champaign (1995)

48. Blumenfeld, W.: Observations concerning the phenomenon and origin of play. Philos. Phenomenol. Res. 1, 470-478 (1941)

49. Champlin, N.L.: Are sports methodic? J. Philos. Sport. 4, 104-116 (1977)

50. Osterhoudt, R.: "Physicality": one among the internal goods of sport. J. Philos. Sport. XXIII, 91-103 (1996)

51. Paddick, R.J.: What makes physical activity physical? J. Philos. Sport. 2, 12-22 (1975)

52. Hsu, L.: Revisiting the concept of sport. J. Humanit. Soc. Sci. 1, 45-54 (2005)

53. Tiedemann, C.: Sport (and culture of physical motion) for historians, an approach to precise the central term(s). In: Teja, A., Krüger, A., Riordan, J. (eds.) Sport and Cultures, Proceedings of the 9th International Congress of the European Committee for Sport History (CESH), Crotone, Italy, pp. 410-416 (2004)

Open Access This chapter is licensed under the terms of the Creative Commons Attribution 4.0 International License (http://creativecommons.org/licenses/by/4.0/), which permits use, sharing, adaptation, distribution and reproduction in any medium or format, as long as you give appropriate credit to the original author(s) and the source, provide a link to the Creative Commons license and indicate if changes were made.

The images or other third party material in this chapter are included in the chapter's Creative Commons license, unless indicated otherwise in a credit line to the material. If material is not included in the chapter's Creative Commons license and your intended use is not permitted by statutory regulation or exceeds the permitted use, you will need to obtain permission directly from the copyright holder.

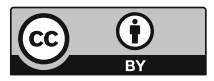

